\title{
"NANGAÑANA" (TREMELLOSCYPHA GELATINOSA, SEBACINACEAE), HONGO SILVESTRE COMESTIBLE DEL BOSQUE TROPICAL DECIDUO EN LA DEPRESIÓN CENTRAL DE CHIAPAS, MÉXICO
}

\author{
Victor Manuel Bandala ${ }^{1,4}$, Leticia Montoya ${ }^{1}$, Rafael Villegas ${ }^{2}$, Teresa \\ Guadalupe Cabrera ${ }^{3}$, Manuel de Jesús Gutiérrez ${ }^{3}$ y Tomás Acero ${ }^{3}$ \\ ${ }^{1}$ Instituto de Ecología, A.C., Red de Biodiversidad y Sistemática, \\ Apdo. postal 63, 91000 Xalapa, Veracruz, México. \\ ${ }^{2}$ Instituto de Ecología, A.C., Red de Ambiente y Sustentabilidad, \\ Apdo. postal 63, 91000 Xalapa, Veracruz, México. \\ ${ }^{3}$ Secretaría de Medio Ambiente e Historia Natural, Dirección de Botánica, \\ Tuxtla Gutiérrez, Chiapas, México. \\ ${ }^{4}$ Autor para la correspondencia: victor.bandala@inecol.mx
}

\section{RESUMEN}

Se presenta información sobre el uso de los esporomas de Tremelloscypha gelatinosa como alimento por los habitantes nativos de Nandayalu, una localidad al SE de Tuxtla Gutiérrez, Chiapas, México. El hongo es localmente llamado "nangañaña", los cuerpos fructíferos se recogen durante parte de la temporada de lluvias (julio-agosto) y se usan para consumo familiar. Se describe el método de recolección y de preparación, y se presentan fotografías de sus basidiomas, de su hábitat y usos. La especie se registró fructificando en el suelo, bajo árboles de Gymnopodium floribundum, en bosque tropical deciduo en la depresión central de Chiapas. Tremelloscypha gelatinosa es un heterobasidiomiceto del orden Sebacinales y su relación filogenética con especies que forman micorrizas sugiere su posible relación ectomicorrízica con las raíces de las plantas y, por tanto, desempeñando una importante función ecológica en los ecosistemas tropicales donde se desarrolla. Es un hongo poco frecuente, hasta ahora registrado de Jamaica (localidad tipo), Florida (E.U.A.), Yucatán y Quintana Roo (México). No se tenía conocimiento de su uso como elemento comestible silvestre.

Palabras clave: etnomicología, hongos comestibles silvestres, Gymnopodium, Polygonaceae. 


\begin{abstract}
We present information on the use of sporomes of Tremelloscypha gelatinosa as a food source among native people from Nandayalu, a locality situated SE of Tuxtla Gutiérrez, Chiapas, Mexico. The fungus is locally named "nangañaña" and its fruit bodies are collected during part of the rainy season (June-August) and are used for familiar consumption. Their methods of collecting and preparation are described and photographs of the basidiomes and habit are provided. Tremelloscypha gelatinosa was found growing below trees of Gymnopodium floribundum in the tropical deciduous forest of the Central Chiapas basin. It is a heterobasidiomycete belonging to the order Sebacinales and its phylogenetic placement among proven ectomycorrhizal sebacinoid species suggests its probable ectomycorrhizal symbiosis with plant roots, thus playing a relevant ecological role in tropical ecosytems where it occurs. Tremelloscypha gelatinosa is an infrequently encountered fungus, currently known from Jamaica (type locality), Florida (USA), Yucatán and Quintana Roo (Mexico) and until now unreported as a wild edible fungus.
\end{abstract}

Key words: ethnomycology, Gymnopodium, Polygonaceae, wilde edible fungi.

\title{
INTRODUCCIÓN
}

La diversidad biológica y cultural propia de México, en su conjunto, han posicionado al país en una escala comparativamente importante a nivel mundial en esos dos escenarios (Mittermeier et al., 1997; Loh y Harmon, 2005). Los hongos comestibles silvestres armonizan perfectamente en esos dos ámbitos, considerando que en México este grupo de productos no maderables del bosque destaca por su diversidad de especies y porque desde la antigüedad tales organismos llamaron la atención de la población autóctona, la que constantemente los incluyó en sus prácticas sociales y/o económicas confrontadas al principio con su medio silvestre, revirtiendo como resultado una sólida historia de conocimiento tradicional en torno a su nomenclatura, ecología, manejo, uso y conservación. Además de la importancia ecológica de los hongos comestibles silvestres, su valor agregado como fuente de alimento ha beneficiado a la población nativa especialmente la del medio rural de México, bien sea por incluirlos en su dieta y/o por obtener un beneficio económico por su venta local (Mapes et al., 1981; Bandala et al., 1997; Montoya-Esquivel, 1998). En términos generales, ese conocimiento es una herencia cultural legada de nuestros antecesores, de manera que actualmente se sabe que en diferentes sitios de distintas entidades 
del territorio mexicano, especialmente en época de lluvias, se recolectan con mayor o menor intensidad, cuerpos fructíferos de varias especies de hongos silvestres para su consumo y venta (Montoya-Esquivel, 1998).

Casos particulares que se apartan quizá del contexto de ese legado cultural, lo constituyen taxones de los que no se tienen evidencias claras de formar parte del paquete básico de especies, ni local ni tradicionalmente utilizadas desde el pasado, pero que sí son catalogadas con valor por algunas otras etnias. Después del registro de Tricholoma magnivelare (Peck) Redhead en México (Montoya et al., 1987, como T. ponderosum (Peck) Singer), por ejemplo, la especie (a la que se le denominó hongo blanco de ocote $\mathrm{u}$ hongo blanco) se adicionó al conocimiento local sobre hongos comestibles silvestres de la región. Este proceso se registró como consecuencia de la catalogación de T. magnivelare dentro del grupo de especies relacionadas con el matsutake asiático (T. matsutake (S. Ito \& S. Imai) Singer) y las implicaciones económicas entorno a este último. Así, T. magnivelare se posicionó automáticamente como un hongo comestible silvestre altamente cotizado y rápidamente se convirtió en objeto de recolección por los beneficios que detectó la población, al no tener este hongo una demanda local pero sí una gran importancia dentro del flujo comercial a escalas internacionales, derivada de la cultura asiática (Villarreal y Pérez-Moreno, 1989: Bandala et al., 1997).

Los habitantes de cada región de México mantienen cierta preferencia por determinadas especies, aunque en los mercados populares de asentamientos ubicados a lo largo del Eje Neovolcánico, es común encontrar en venta a Amanita caesarea (Scop.) Pers., A. rubescens Pers., Boletus edulis Bull., Cantharellus cibarius Fr., Lactarius indigo (Schwein.) Fr., Morchella esculenta (L.) Pers., Russula brevipes Peck, y Ustilago maydis (DC.) Corda, entre muchas otras (Herrera y Guzmán, 1961; Mapes et al., 1981; Bandala et al., 1997; Montoya et al., 2003). En el bosque tropical deciduo de la depresión central de Chiapas, cerca de Tuxtla Gutiérrez (sur de México), encontramos fructificando en el suelo del sotobosque de Gymnopodium floribundum Rolfe (Polygonaceae Juss.) a Tremelloscypha gelatinosa (Murrill) Oberw. \& K. Wells (Bandala et al., 2011). Descubrimos que los esporomas de esta especie son usados como alimento para consumo familiar. En la literatura encontramos pocos registros y especímenes de esta especie y hasta ahora no se tiene información del valor agregado que tiene el mencionado hongo al tratarse de una especie silvestre comestible, razón por la cual elaboramos el presente trabajo. Tremelloscypha gelatinosa se ha citado de Jamaica (localidad tipo), E.U.A. (Florida) y México (Yucatán y Quintana Roo) (Burt, 1915 como Eichleriella, Wells, 1961; Wells y Oberwinkler, 1982; Guzmán, 2004; Roberts, 2006). 
Es un hongo que durante sus fases de desarrollo produce cuerpos fructíferos que varían desde pulvinados y sésiles hasta rudimentariamente estipitados y pseudoinfundibiliformes, robustos, con textura coriácea, fibrosos y de consistencia esponjosa, por lo que tienen aspecto de algunas formas de hongos poliporáceos, estereáceos o gomfáceos. Su himenóforo es decurrente, liso, con aspecto cartilaginoso y produce heterobasidios. Crece en el suelo, entre la hojarasca y otros restos vegetales (Bandala et al., 2011). Presentamos la información que conocimos en la comunidad de Nandayalu, municipio de Suchiapa, Chiapas, sobre la recolección, preparación y usos de T. gelatinosa, así como fotografías de los basidiomas y otros aspectos en su entorno ecológico y culinario.

\section{MÉTODOS}

Área de estudio. La información obtenida y los hongos recolectados procede de la comunidad de Nandayalu, municipio de Suchiapa, SE de Tuxtla Gutiérrez, ubicada en la depresión central del estado de Chiapas (México). Esta región está caracterizada por presentar actualmente varias hectáreas irregularmente cubiertas por asociaciones dominadas o co-dominadas por árboles de Gymnopodium floribundum, que forma parte del bosque tropical deciduo establecido en la depresión central de Chiapas (Miranda, 1952; Reyes-García y Souza, 1997). Se ubica a una altitud de $460 \mathrm{~m}$, cuenta con una precipitación promedio que fluctúa entre $900 \mathrm{y}$ $1200 \mathrm{~mm}$ recibida principalmente de mayo a octubre y con suelo mayormente derivado de caliza.

Recolección, estudio taxonómico de muestras y recopilación de información sobre su consumo. Se recolectó material fresco en diferentes visitas efectuadas al área antes mencionada, entre junio-septiembre y noviembre, durante 2003-2008. Para todo lo relacionado con la identificación taxonómica de la especie referimos al trabajo de Bandala et al. (2011). Los especímenes estudiados forman parte del Herbario XAL. Durante las visitas y en diferentes períodos, se efectuaron observaciones directas y entrevistas entre varios informantes del lugar para la recopilación de datos sobre la temporada de fructificación de T. gelatinosa, ecología, sitios en el bosque donde aparecen las fructificaciones, nomenclatura autóctona, identificación del género y edad de los participantes en la recolección del hongo, estrategia de recolección y proceso de preparación para consumo. Dos de los autores (M.J. Gutiérrez y T. Acero), originarios de la localidad, practicaron la recolección de fructificaciones para cocinarlas. 


\section{RESULTADOS}

La práctica de la recolección de esporomas de T. gelatinosa es resultado de un arraigado conocimiento tradicional entre la población nativa de la zona. Actualmente se efectúa durante parte de la temporada de lluvias, entre julio y septiembre; en julio y a mediados de agosto es cuando se observa el mayor número de fructificaciones (Fig. 2a). En el bosque de Gymnopodium floribundum visitado también está presente otra especie de hongo comestible llamada localmente "moni" (Miranda, 1952) que corresponde a Lactarius chiapanensis Montoya, Bandala \& Guzmán (Montoya et al., 1996) y la cual comparte con T. gelatinosa casi el mismo patrón fenológico de basidiomas. En términos de ubicación espacial de cuerpos fructíferos de T. gelatinosa, ésta parece estar relacionada con la aparente asociación simbiótica Gymnopodium floribundum - Tremelloscypha gelatinosa. La marcada presencia de T. gelatinosa bajo los árboles de Gymnopodium quizá sea un indicador de que formen algún tipo de micorriza. Bajo el mismo esfuerzo de muestreo y al igual como se observó con las fructificaciones de L. chiapanensis, todos los especímenes de $T$. gelatinosa se encontraron en grupos dispersos en el suelo del bosque, pero siempre en la cercanía de individuos de G. floribundum (Fig. la-e). Reconocimos que ambas especies de hongos son las que hasta ahora tienen la mayor importancia cultural entre los habitantes de esta región de Chiapas. Los esporomas se recolectan simultáneamente para consumo, pero dando preferencia a los del "moni" que son objeto de venta en los mercados populares de la región o aún en los mercados de Tuxtla Gutiérrez. Los informantes mencionaron que las fructificaciones de L. chiapanensis, al tener un sabor y textura (no correosa) más agradable que las de $T$. gelatinosa, así como su aspecto (sombrero, láminas y estípite) se valoran por la población rural y urbana como los típicos hongos para venta en los mercados, y por ser aparentemente más abundantes se comercializan a esa escala.

Los árboles de G. floribundum en la región de Suchiapa, son conocidos como "nangañas" o "nangaños" (de la lengua chiapaneca "na'ngaña") y colectivamente la asociación vegetal es llamada "nangañal", lo cual es equivalente al "aguanal" o "aguanacatonal" citado por Miranda (1952) como asociaciones de G. antigonoides (B.L. Rob. ex Millsp. \& Loes.) S.F. Blake. El sitio dentro del municipio de Suchiapa se conoce como Nandayalu en lengua chiapaneca, que significa paraje, arroyo de alcaravanes, del sustantivo "nanda" equivalente a arroyo, río, riachuelo, paraje cerca del río (Aguilar, 1992), y hace referencia justamente a la ubicación del asentamiento de los pobladores a las orillas de un arroyo. La aparente estrecha relación ecológica de T. gelatinosa con las "nangañas" propicia la frecuente presencia de esporomas en 
Acta Botanica Mexicana 106: 149-159 (2014)
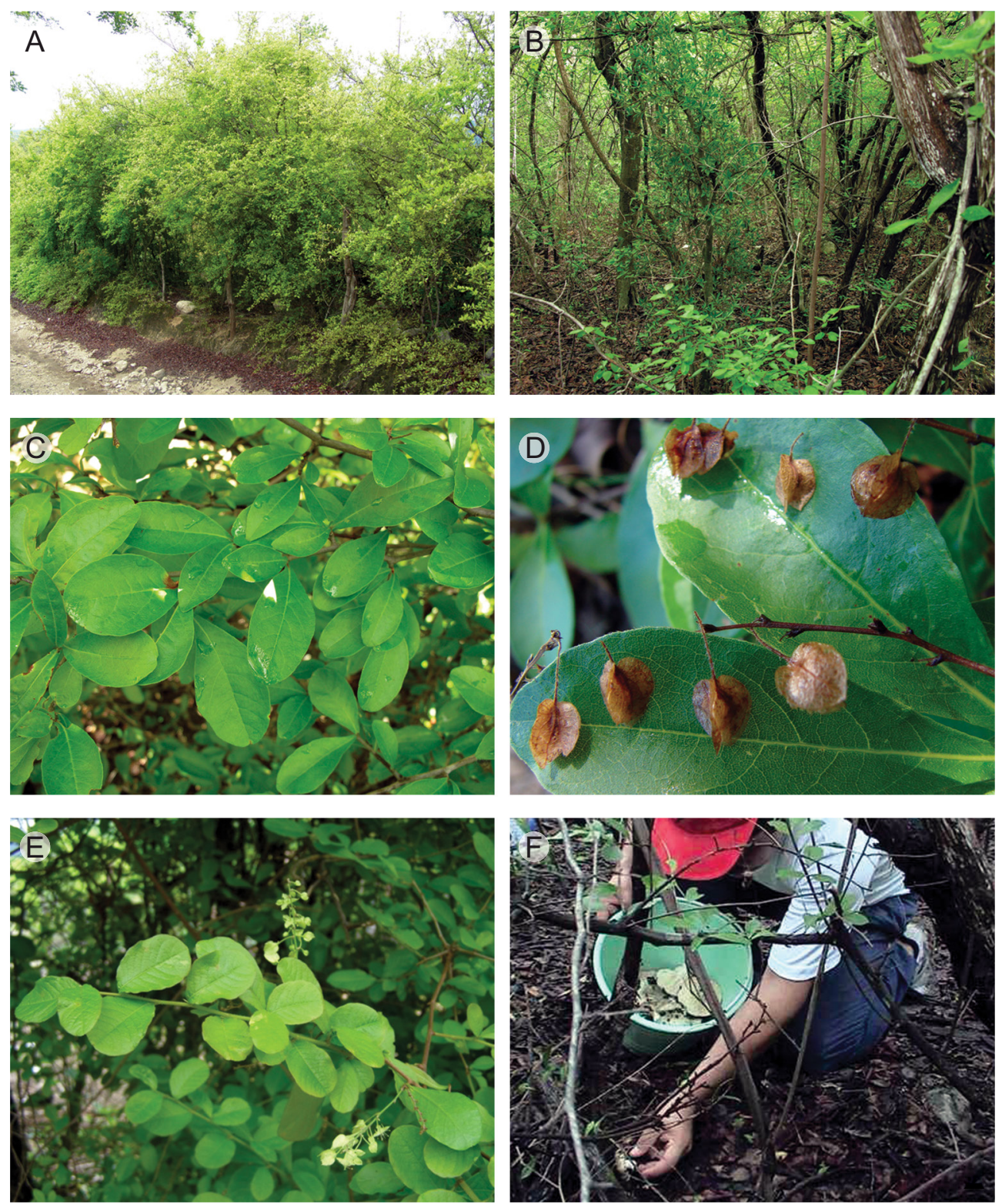

Fig. 1. A-B. Bosque de Gymnopodium floribundum; C-E. detalle de hojas, frutos y flores de plantas de G. floribundum; F. recolecta de esporomas de nangañaña (Tremelloscypha gelatinosa) y moni (Lactarius chiapanensis). 
el sotobosque, razón por la que los habitantes del lugar asocian a estos hongos con ese tipo de vegetación. Como "na'ngaña" es el nombre de las plantas de G.floribundum, "na'ngañaná" (o en castellano "nangañaña") es el nombre con el que los habitantes del lugar denominan a las fructificaciones de T. gelatinosa y lo que en lengua chiapaneca se refiere a "hongo comestible de las nangañas". En la recolección de basidiomas de "nangañaña" participan todos los integrantes de una familia (padre, madre e hijos de distintas edades), cada uno con su propia habilidad para efectuar la búsqueda y obtención de fructificaciones (Fig. 1f). Se debe mencionar que los elementos arbóreos de G. floribundum en los sitios visitados, en cortas distancias muestran variación tanto en densidad por el número de árboles, como por la arquitectura de su copa, encontrándose áreas con individuos con diferencia de tamaño, de tipo de crecimiento y de número/tamaño de ramificaciones (más o menos similares a las formas arbóreas descritas por Interián-Ku et al., 2009). Esta condición dificulta la movilidad dentro del bosque, ya que prácticamente la persona efectúa su desplazamiento en forma inclinada, quizá motivo por el cual particularmente los hombres y mujeres jóvenes son los que mejor efectúan la recolección de hongos con zigzagueos a través de los árboles. Los recorridos para la obtención de hongos inician temprano por la mañana dirigiéndose la familia al bosque con recipientes de plástico (cubetas o botes), donde van depositando las fructificaciones de los hongos extraídas del suelo con la ayuda de un machete o bien manualmente. No existe preferencia por el estado de desarrollo de las fructificaciones y cualquier espécimen es objeto de recolección. La jornada termina cuando consideran que han colectado lo suficiente para preparar el alimento para toda la familia. No conocimos alguna forma de preservación de los hongos, ya que se cocinan inmediatamente después de su recolección. Son parte de su dieta como uno más de los recursos silvestres no maderables del bosque, disponibles por temporada, y que los habitantes del sitio claramente reconocen dentro del concepto de hongo y no de planta.

En virtud de la consistencia correosa de las fructificaciones de T. gelatinosa, el método tradicional de preparación es en combinación con memelas (tortilla gruesa hecha a base de masa de maíz y doblada) (Fig. 2b-f), lo que significa que intervienen dos principales elementos naturales para la elaboración del alimento, los hongos y el maíz, siendo este último un recurso utilizado en todo México para la preparación de nixtamal (granos de maíz cocidos en agua con cal) y subsecuentemente molido para producir la masa con la que se elaboraran las tortillas (Massieu y Montenegro, 2002). Las fructificaciones de $T$. gelatinosa se limpian con agua corriente y después se deshebran manualmente o se cortan en tiras. Debido a su consistencia, se muelen en un molinillo manual, donde simultáneamente se adiciona chile jalapeño (una 

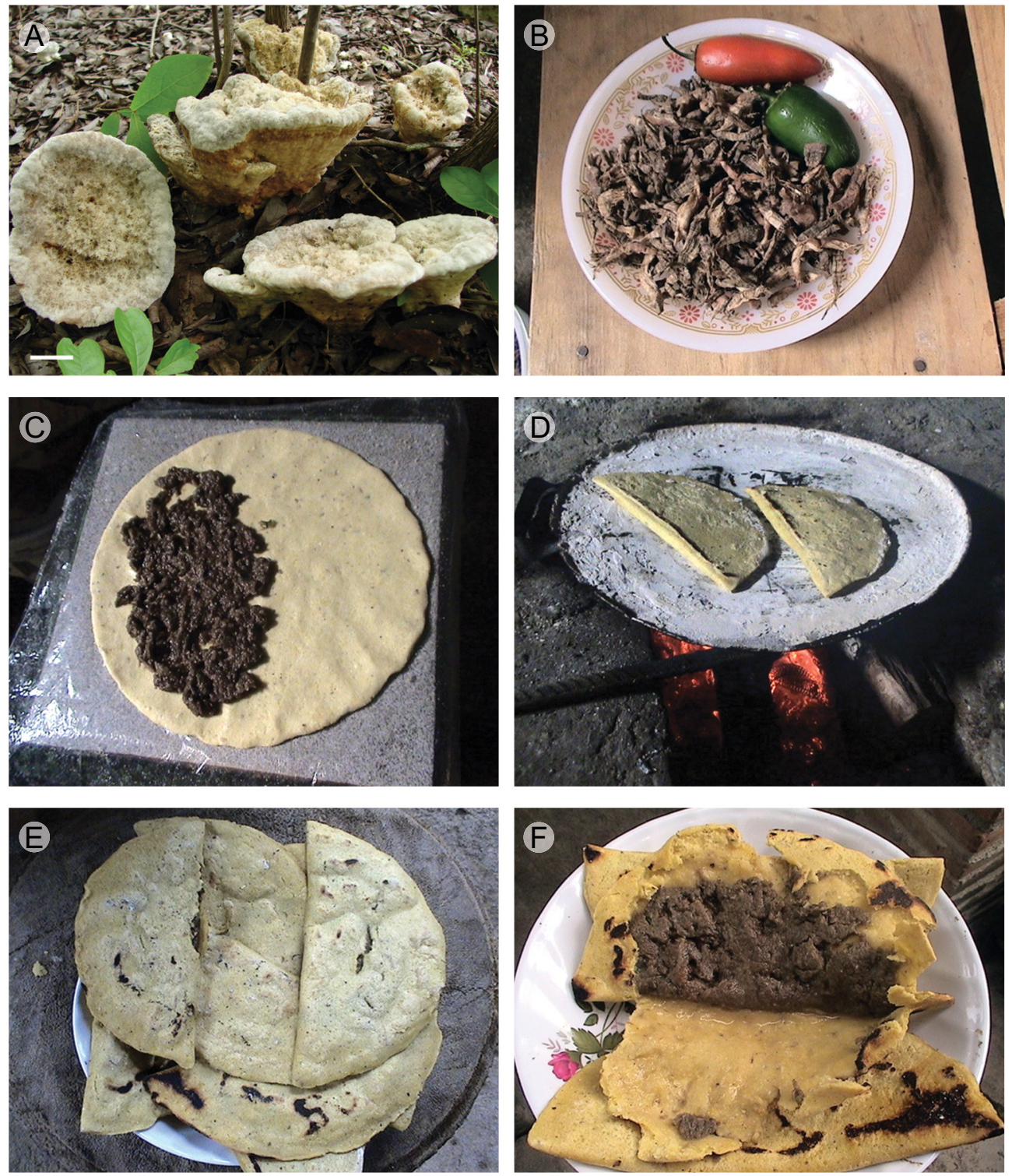

Fig. 2. A. Basidiomas de Tremelloscypha gelatinosa (escala=2cm); B. hongo deshebrado y cortado, acompañado de chile jalapeño; C. pasta del hongo y chile molido sobre tortilla de masa de maíz elaborada a mano; D. cocimiento de la memela sobre un comal; E-F. memelas cocidas listas para consumo. 
variedad de Capsicum annuиm L.) a fin de realizar una mezcla de masa homogénea de chile-hongo con algo de sal, que servirá para el relleno de la memela. Una vez elaborada la tortilla de maíz, se coloca sobre ella la mencionada masa del hongo y chile, se dobla y se coloca en el comal para su cocción (Fig. 2b-f).

\section{DISCUSIÓN}

El hallazgo de T. gelatinosa en la localidad de Nandayalu en Chiapas, México, nos revela dos importantes aspectos de su valor cultural y ecológico. El primero es referente a que en nuestro país todavía existen especies de hongos que forman parte del legado cultural, los cuales durante años han sido conocidos y consumidos localmente por los habitantes de sitios particulares, y solo recientemente registrados en el ámbito científico para incorporarse al catálogo nacional. Pueden citarse, además de T. gelatinosa, los casos de L. chiapanensis de la región zoque de Tuxtla Gutiérrez, Chiapas (Montoya et al., 1996), Neolentinus ponderosus (O.K. Mill.) Redhead \& Ginns de la zona tarahumara de Chihuahua (Moreno-Fuentes et al., 1996), Calostoma cinnabarinum Corda de la región sierra Otomí-Tepehua en Hidalgo (Bautista-Nava y MorenoFuentes, 2009) y Ramaria araiospora var. araiospora Marr \& D.E. Stuntz, R. celerivirescens Marr \& D.E. Stuntz, y Gomphus kauffmanii (A.H. Sm.) Corner del noreste del Estado de México con influencia otomí y mazahua (Aguilar y Villegas, 2010).

El otro aspecto es la relevancia de la presencia de $T$. gelatinosa en el "nangañal", ya que los individuos de esta especie de heterobasidiomicete podrían desempeñar una importante función ecológica en caso de establecer asociación a través de algún tipo de micorríza con las raíces de plantas de G. floribundum o de algunas otras especies en el bosque de esa región de Chiapas. Esta consideración se basa en el íntimo parentesco filogenético de $T$. gelatinosa con especies que forman ectomicorrizas y que pertenecen al orden Sebacinales M. Weiß, Selosse, Rexer, A. Urb. \& Oberw. (grupo A) (Weiß y Oberwinkler, 2001; Selosse et al., 2002; Weiß et al., 2004). De hecho, el "nangañal" alberga una comunidad de hongos ectomicorrizógenos, ya que durante nuestros recorridos registramos varios especímenes de los géneros Amanita, Cantharellus, Inocybe, Lactarius y Russula, entre otros (algunos representan especies comestibles silvestres que igualmente se incluyen en el mencionado concepto de "moni"), lo que podría considerarse como un buen indicador del valor ecológico del sistema de redes de micorrizas bajo los árboles de G. floribundum y de la importancia de esta especie arbórea que domina en la región formando asociaciones que cubren extensiones relativamente amplias aunque actualmente fragmentadas. 


\section{AGRADECIMIENTOS}

Reconocemos las facilidades prestadas durante las visitas, recorridos, apoyo logístico en el campo y preparación de "nangañaña" a las familias Gutiérrez Espinoza, Espinoza Vicente y Espinoza Clemente de la comunidad de Nandayalu. También a la Sra. Romelia Cundapi por permitirnos explorar su propiedad cubierta con bosque de G. floribundum. Agradecemos la asistencia en el laboratorio al biólogo D. Ramos (Instituto de Ecología, Xalapa).

\section{LITERATURA CITADA}

Aguilar, M. 1992. Diccionario de la lengua chiapaneca. Porrúa-Gobierno del Estado de Chiapas. México, D.F. 784 pp.

Aguilar, Y. y M. Villegas. 2010. Especies de Gomphales comestibles en el municipio de Villa del Carbón, Estado de México. Rev. Mex. Mic. 31: 1-8.

Bandala, V. M., L. Montoya y I. Chapela. 1997. Wild edible mushrooms in Mexico: a challenge and opportunity for sustainable development. In: Palm, M. y I. Chapela (eds.). Mycology in sustainable development: expanding concepts, vanishing borders. Parkway, USA. pp. 76-90.

Bandala, V. M., L. Montoya y R. Villegas. 2011. Tremelloscypha gelatinosa (Sebacinales) occurring in Gymnopodium forests in the tropical deciduous vegetation from southern Mexico. Mycotaxon 118: 147-157.

Bautista-Nava, E. y A. Moreno-Fuentes. 2009. Primer registro de Calostoma cinnabarina (Sclerodermatales) como especie comestible. Rev. Mex. Biodiv. 80: 561-564.

Burt, E. A. 1915. The Thelephoraceae of North America V. Tremellodendron, Eichleriella and Sebacina. Ann. Missouri Bot. Gard. 2: 731-771.

Guzmán, G. 2004. Los hongos de la Península de Yucatán (México) V. Nuevas observaciones y nuevos registros. Rev. Mex. Mic. 18: 7-13.

Herrera, T. y G. Guzmán. 1961. Taxonomía y ecología de los principales hongos comestibles de diversos lugares de México. Anales Instituto de Biología, Ser. Bot. 22: 33-135.

Interián-Ku, V. M., J. I. Valdez-Hernández, E. García-Moya, A. Romero-Manzanares, M. A. Borja de la Rosa y H. Vaquera-Huerta. 2009. Arquitectura y morfometría de dos especies arbóreas en una selva baja caducifolia del sur de Yucatán, México. Bol. Soc. Bot. Méx. 85: 17-29.

Loh, J. y D. Harmon. 2005. A global index of biocultural diversity. Ecological Indicators 5: 231-24.

Mapes, C., G. Guzmán y J. Caballero. 1981. Etnomicología purépecha: El conocimiento y uso de los hongos en la Cuenca del Lago de Pátzcuaro, Michoacán. Serie Etnociencia 2. Dirección General de Culturas Populares (Secretaría de Educación Pública) y Sociedad Mexicana de Micología, A.C. Instituto de Biología, Universidad Nacional Autónoma de México. México, D.F. p. 79. 
Massieu, Y. y J. L. Montenegro. 2002. El maíz en México: biodiversidad y cambios en el consumo. Rev. Anál. Económico 36: 281-303.

Miranda, F. 1952. La vegetación de Chiapas. Ediciones del Gobierno de Chiapas. Tuxtla Gutiérrez, México. 600 pp.

Mittermeier, R. A., P. Robles-Gil y C. G. Mittermeier. 1997. Megadiversity. CEMEX Mexico, D.F., México. 503 pp.

Montoya, A., O. Hernández-Totomoch, A. Estrada-Torres, A. Kong y J. Caballero. 2003. Traditional knowledge about mushrooms in a Nahua community in the state of Tlaxcala, México. Mycologia 95: 793-806.

Montoya, L., V. M. Bandala y G. Guzmán. 1987. Nuevos registros de hongos del estado de Veracruz, IV. Agaricales II (con nuevas colectas de Coahuila, Michoacán, Morelos y Tlaxcala). Rev. Mex. Mic. 3: 83-107.

Montoya, L., V. M. Bandala y G. Guzmán. 1996. New and interesting species of Lactarius from Mexico, including scanning electron microscope observations. Mycotaxon 57: 411-424.

Montoya-Esquivel, A. 1998. Ethnomycology of Tlaxcala, Mexico. McIlvainea 13(2): 6-12.

Moreno-Fuentes, A., J. Cifuentes, R. Bye y R. Valenzuela. 1996. Kute-mo'ko-a: un hongo comestible de los indios Raramuri de México. Rev. Mex. Mic. 12: 31-39

Reyes-García, A. y M. Sousa. 1997. Listados florísticos de México XVII. Depresión Central de Chiapas. La selva baja caducifolia. Instituto de Biología, Universidad Nacional Autónoma de México. México, D.F., México. 47 pp.

Roberts, P. 2006. Caribbean heterobasidiomycetes: 2. Jamaica. Mycotaxon 96: 83-107.

Selosse, M. A., M. Weiß, J. L. Jany y A. Tillier. 2002. Communities and populations of sebacinoid basidiomicetes associated with the achlorophyllous orchid Neottia nidusavis (L.) L.C.M. Rich. and neighbouring tree ectomycorrhizae. Mol. Ecol. 11: 18311844.

Villarreal, L. y J. Pérez-Moreno. 1989. Aprovechamiento y conservación del matsutake americano (Tricholoma magnivelare) en los bosques de México. Micología Neotropical. Apl. 2: 131-144.

Weiß, M. y F. Oberwinkler. 2001. Phylogenetic relationships in Auriculariales and related groups-hypotheses derived from nuclear ribosomal DNA sequences. Mycol. Res. 105: 403-415.

Weiß, M., M. A. Selosse, K. H. Rexer, A. Urban y F. Oberwinkler. 2004. Sebacinales: a hitherto overlooked cosm of heterobasidiomycetes with a broad mycorrhizal potential. Mycol. Res. 108: 1003-1010.

Wells, K. 1961. Studies of some Tremellaceae IV. Exidiopsis. Mycologia 53: 317-370.

Wells, K. y F. Oberwinkler. 1982. Tremelloscypha gelatinosa, a species of a new family Sebacinaceae. Mycologia 74: 325-331. 